\title{
Nickel nanoparticles in hydrogen-transfer reductions: Characterisation and nature of the catalyst
}

\author{
Francisco Alonso*, Paola Riente, Juan Alberto Sirvent, Miguel Yus* \\ Departamento de Química Orgánica, Facultad de Ciencias, and Instituto de Síntesis Orgánica (ISO), Universidad de Alicante, Apdo. 99, 03080 Alicante, Spain
}

\section{A R T I C L E I N F O}

\section{Article history:}

Received 2 October 2009

Received in revised form 21 January 2010

Accepted 29 January 2010

Available online xxx

Dedicated to Professor Pelayo Camps on

occasion of his 65th birthday.

\section{Keywords:}

Hydrogen transfer

Heterogeneous catalysis

Nickel nanoparticles

\begin{abstract}
A B S T R A C T
Nickel nanoparticles, readily prepared by reduction of nickel(II) chloride with lithium and a catalytic amount of DTBB, have been used in the transfer hydrogenation of carbonyl compounds and have been fully characterised by different means. The reaction rate of the transfer hydrogenation was found to be dependent on the acetophenone and isopropanol concentration but independent on the amount of lithium chloride. The deactivation of the catalyst after reuse has been mainly attributed to surface oxidation but not to coke formation. All the experiments performed are in agreement with the process being of heterogeneous nature. The nickel nanoparticles unveiled a superior behaviour in comparison with commercially available nickel catalysts.
\end{abstract}

(c) 2010 Elsevier B.V. All rights reserved.

\section{Introduction}

In the recent years, considerable attention has been devoted to the development of uniform nanometre-sized nickel nanoparticles because of their unique properties and potential applications in a variety of fields including electronics [1], magnetism [2], energy technology [3], or biomedicine [4]. In comparison with the noble metals, nickel nanoparticles have been much less studied in catalysis, although they have found a particular application in the growth of carbon nanotubes [5] as well as in a variety of organic reactions [6]. The synthesis of nickel nanoparticles in the zerovalence state is not trivial since they readily undergo oxidation, consequently affecting their catalytic performance [7]. Nickel nanoparticles are mostly synthesised by the chemical reduction of a nickel(II) salt, with the polyol process [8] and hydrazine [9] or sodium borohydride [10] reduction being the most practiced methods. In general, the presence of an additive, as protective agent, is necessary and a common feature in all these methodologies in order to prevent particle agglomeration.

As part of our continuous interest on the preparation and application of active metals [11], we reported the fast synthesis of nickel(0) nanoparticles (NiNPs), from different nickel(II) chloridecontaining systems in THF, using lithium powder and a catalytic amount of an arene as reducing agent [12]. The method was

\footnotetext{
* Corresponding authors. Tel.: +34 965903548; fax: +34 965903549

E-mail addresses: falonso@ua.es (F. Alonso), yus@ua.es (M. Yus).
}

applied in the absence of any anti-agglomeration additive or nucleation catalyst at room temperature. These nanoparticles found application in different functional group transformations [13] as well as in the hydrogen-transfer reduction of carbonyl compounds and olefins [14], and reductive amination of aldehydes [15]. We also discovered that nickel, in the form of nanoparticles, can activate alcohols for the $\alpha$-alkylation of ketones and indirect aza-Wittig reaction, with this being a potential alternative to noble-metal-based methodologies [16]. These reactions involved the hydrogen transfer from the alcohol to the intermediate $\alpha, \beta$ unsaturated ketone or imine, respectively. Moreover, in contrast with the use of noble-metal catalysts, the reactions proceeded in the absence of any added ligand, hydrogen acceptor or base, under mild conditions. Very recently, we have demonstrated for the first time that nickel, in the form of nanoparticles, can promote the Wittig-type reaction of primary alcohols and phosphorus ylides [17]. Moreover, a series of polymethoxylated stilbenes as well as resveratrol, DMU-212, and analogues, have been synthesised using this novel Wittig-type olefination as the key step.

It is noteworthy that the NiNPs were shown to be catalytically superior to other forms of nickel in all the aforementioned reactions. Of particular interest is their application to the catalytic transfer hydrogenation with isopropanol of carbonyl compounds, where the NiNPs could be reutilised several times maintaining a high activity in a very simple reaction medium composed of NiNPs, isopropanol and the substrate, in the absence of any base [14]. A detailed characterisation of the NiNPs in this reaction medium, however, has not been yet tackled. We wish to report herein a 
complete study on the characterisation of the NiNPs, utilised in transfer hydrogenation reactions with isopropanol as the hydrogen donor, as well as a series of catalytic and kinetic experiments. Since catalysis by metal nanoparticles can be considered a kind of "semiheterogeneous catalysis", at the frontier between homogeneous and heterogeneous catalysis [18], the present study will also include a series of tests in order to determine the catalytic nature of the process.

\section{Experimental}

\subsection{General}

Dry THF was directly used without any purification (Fluka, 99.9\%). Anhydrous nickel(II) chloride (Alfa Aesar, 98\%), lithium powder (MEDALCHEMY S. L.), 4,4'-di-tert-butylbiphenyl (DTBB, Fluka), isopropanol (Panreac, 99.8\%), acetophenone (Aldrich), and triphenylphosphane (Aldrich) were commercially available of the best grade and were used without further purification. The chromatographic analyses (GLC) were performed with a Hewlett Packard HP-6890 instrument equipped with a flame ionisation detector and a $30 \mathrm{~m} \mathrm{HP}-1$ capillary column [0.32 mm diameter, $0.25 \mu \mathrm{m}$ film thickness (100\% dimethylpolysiloxane)], using nitrogen $(2 \mathrm{~mL} / \mathrm{min})$ as carrier gas, $T_{\text {injector }}=275^{\circ} \mathrm{C}, T_{\text {column }}=60^{\circ} \mathrm{C}$ (3 min) and $60-270{ }^{\circ} \mathrm{C}\left(15^{\circ} \mathrm{C} / \mathrm{min}\right)$.

\subsection{Preparation of the catalyst}

Nickel (II) chloride (130 mg, $1 \mathrm{mmol}$ ) was added over a suspension of lithium (14 mg, $2 \mathrm{mmol}$ ) and DTBB (13 mg, $0.05 \mathrm{mmol})$ in dry THF $(2 \mathrm{~mL})$ at room temperature under argon. The reaction mixture, which was initially dark blue, rapidly changed to black (ca. $5 \mathrm{~min}$ ) indicating that the nickel $(0)$ nanoparticles were formed. Additional stirring of the resulting suspension for at least $10 \mathrm{~min}$ is recommended before use.

\subsection{Characterisation of the catalyst}

$i$-PrOH $(4 \mathrm{~mL})$ was added to the aforementioned NiNPs suspension and the mixture was warmed at $76{ }^{\circ} \mathrm{C}$ during $1 \mathrm{~h}$ (unless otherwise stated).

\subsubsection{Transmission electron microscopy}

TEM images were recorded using a JEOL JEM2010 microscope, equipped with a lanthanum hexaboride filament, operated at an acceleration voltage of $200 \mathrm{kV}$. A drop of the nickel nanoparticle suspension was added to a holey-carbon coated 300 mesh copper grid allowing the solvent to evaporate before being introduced into the microscope. X-EDS analyses were carried out with an Oxford Inca Energy TEM100 attachment.

\subsubsection{X-ray photoelectron spectroscopy}

The XPS spectra were measured with a VG-Microtech Multilab 3000 electron spectrometer using a non-monochromatised $\mathrm{Mg}-\mathrm{K} \alpha$ $(1253.6 \mathrm{eV})$ radiation source of $300 \mathrm{~W}$ and a hemispheric electron analyser equipped with 9 channeltron electron multipliers. The pressure inside the analysis chamber during the scans was about $5 \times 10^{-7} \mathrm{~N} \mathrm{~m}^{-2}$. After the survey spectra were obtained, higher resolution survey scans were performed at pass energy of $50 \mathrm{eV}$. The intensities of the different contributions were obtained by means of the calculation of the integral of each peak, after having eliminated the baseline with $\mathrm{S}$ form and adjusting the experimental curves to a combination of Lorentz (30\%) and Gaussian (70\%) lines. All the bond energies were referred to the line of the $C 1 \mathrm{~s}$ to $284.4 \mathrm{eV}$, obtaining values with a precision of $\pm 0.2 \mathrm{eV}$. The sample was handled under a helium atmosphere unless otherwise stated.

\subsubsection{X-ray powder diffraction}

The XRD diagrams were collected in the $\theta-\theta$ mode using a Bruker D8 Advance X-ray diffractometer: $\mathrm{Cu} \mathrm{K} \alpha_{1}$ irradiation, $\lambda=1.5406 \AA$; room temperature $\left(25^{\circ} \mathrm{C}\right) ; 2 \theta=4-80$. The samples were dried under vacuum and kept under an argon atmosphere.

\subsubsection{Electron paramagnetic resonance}

EPR spectra were recorded on a Bruker EMX EPR spectrometer equipped with a Bruker ER041X microwave bridge X-VAN, operating at X-band frequency $(v=9.8 \mathrm{GHz})$. The samples were diluted with $0.5 \mathrm{~mL}$ of dry 2-propanol under an argon atmosphere and the spectra recorded at room temperature.

\subsubsection{Surface area}

The surface area was measured by the BET (Brunauer-EmmettTeller) method using $\mathrm{N}_{2}$ at $77 \mathrm{~K}$ on a vacuum volumetric gas sorption Autsorb-6 and Autosorb Degasser apparatus (Quantachrome). The sample was carefully filtered through filter paper and dried under vacuum prior to analysis.

\subsubsection{Thermal programmed oxidation}

TPO experiments were carried out in a U-shaped quartz reactor, using a $3 \% \mathrm{O}_{2} / \mathrm{He}$ gas flow of $40 \mathrm{~cm}^{3} / \mathrm{min}$. The sample was subjected to a thermal treatment at a constant heating rate $\left(10^{\circ} \mathrm{C} /\right.$ min), and oxygen consumption and $\mathrm{CO}_{2}$ evolved were monitored as a function of temperature by on-line mass spectrometry (OmniStar system, from Pfeiffer). The sample was previously filtered, washed with isopropanol and ultra-pure water, dried under vacuum for $24 \mathrm{~h}$ and kept under an argon atmosphere.

\subsubsection{Raman spectroscopy}

Raman spectra were recorded on a $50 \mathrm{~mW}$ LabRam spectrometer (Jobin-Ivon Horiba) equipped with a confocal microscope and three laser excitation lines $(\lambda=514,632$, and $785 \mathrm{~nm})$. Detection was done with a CCD detector $(1064 \times 256$ pixels $)$ refrigerated with a Peltier system. The sample was filtered and dried under vacuum prior to analysis.

\subsubsection{Thermogravimetric analysis}

TGA was performed on a TG-DTA apparatus (Mettler Toledo TGA/SDTA851e/LF/1600) connected to a cuadrupolar mass spectrometer (Pfeiffer Vacuum Thermostar GSD301T). The sample was filtered and dried under vacuum prior to analysis.

\subsubsection{Inductively coupled plasma mass spectrometry}

ICP-MS analyses were carried out on a Thermo Elemental VG PQ-ExCell apparatus. The filtrate was evaporated and dissolved with $6 \mathrm{~mL}$ of ultra-pure water.

\subsection{Catalytic activity tests}

\subsubsection{Transfer hydrogenation of acetophenone}

Unless otherwise stated, $i$-PrOH $(4 \mathrm{~mL})$ and acetophenone ( $1 \mathrm{mmol}$ ) were consecutively added to a suspension of the asprepared NiNPs. The reaction mixture was warmed to the indicated temperature and stirred for the specified time. The resulting suspension was diluted with diethyl ether $(20 \mathrm{~mL})$, filtered through a pad containing Celite, and the filtrate was dried over $\mathrm{MgSO}_{4}$ and analysed by GLC. For kinetic studies, the course of the reaction was monitored by GLC analysis of aliquot samples extracted at the specified time.

\subsubsection{Poisoning tests}

$\mathrm{Hg}(0)$ ( 5 or $10 \mathrm{mmol})$ or $\mathrm{PPh}_{3}(0.25,0.5$ or $1 \mathrm{mmol})$ were added over a suspension of the NiNPs $(0.2$ or $1 \mathrm{mmol})$ in the presence of $i$ $\mathrm{PrOH}$ ( $4 \mathrm{~mL})$, followed by the addition of acetophenone $(1 \mathrm{mmol})$. 
(a)

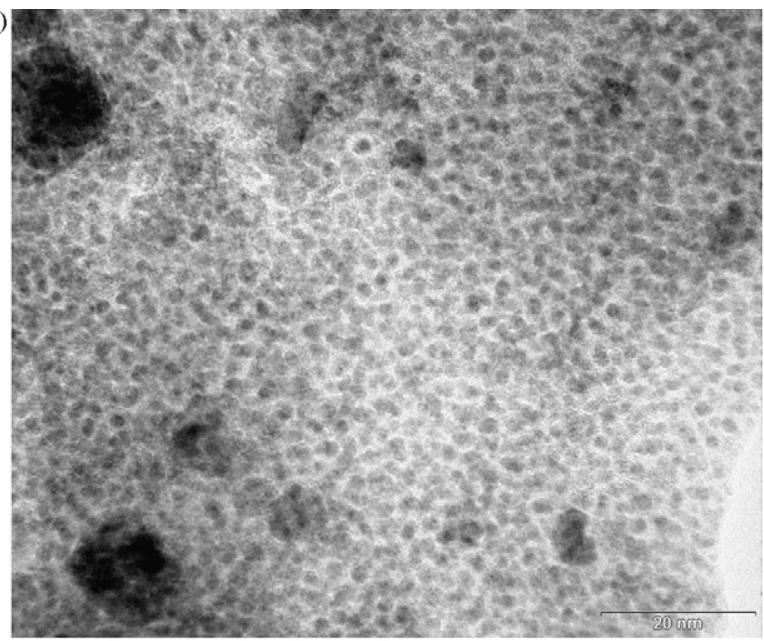

(c)

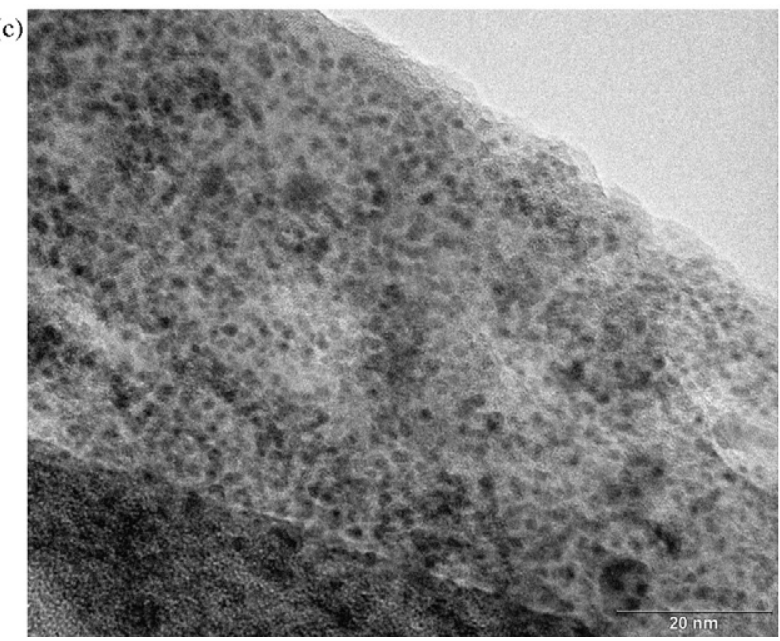

(b)

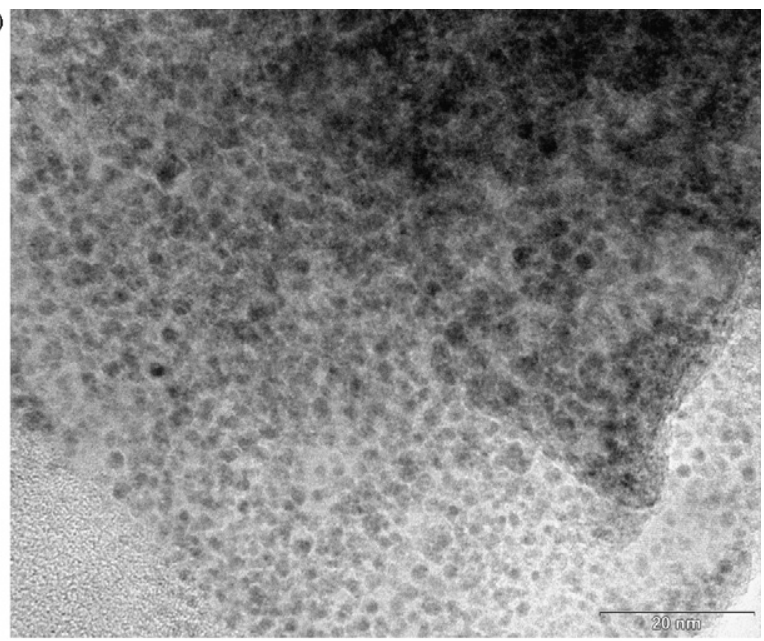

(d)

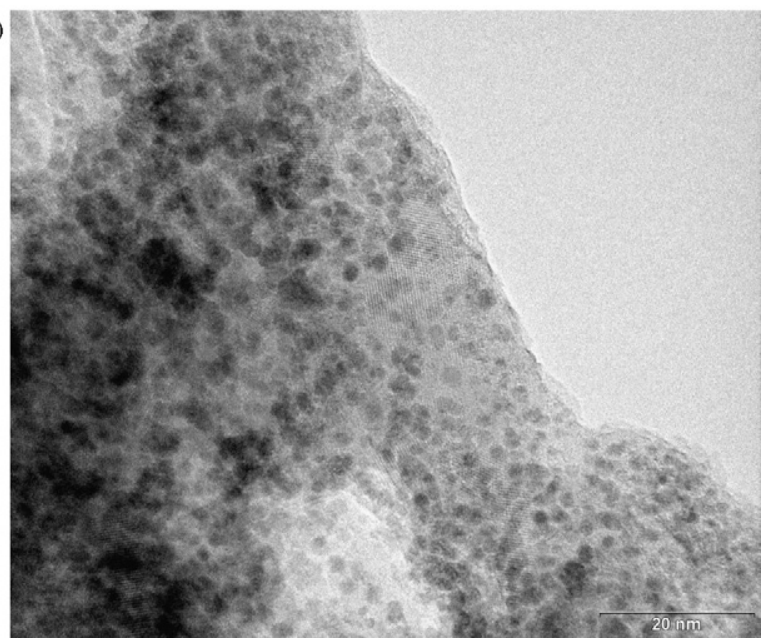

Fig. 1. TEM micrographs of the NiNPs recorded at $1 \mathrm{~h}$ (a), $4 \mathrm{~h}$ (b), $8 \mathrm{~h} \mathrm{(c),} \mathrm{and} 24 \mathrm{~h}$ (d).

The reaction mixture was warmed to $76{ }^{\circ} \mathrm{C}$, stirred at that temperature for $24 \mathrm{~h}$, and worked-up and monitored as said above.

\section{Results and discussion}

\subsection{Catalyst characterisation}

\subsubsection{TEM and EDX}

Droplets of the suspension containing the nickel nanoparticles were analysed at different reaction times (Fig. 1). A typical TEM micrograph and size distribution graphic after $1 \mathrm{~h}$ are depicted in Figs. 1 and 2, respectively. Spherical, monodisperse, and highly uniform nanoparticles were obtained with a narrow range of particle size $(0.75-2.88 \mathrm{~nm}$, ca. $1.75 \pm 1.00 \mathrm{~nm})$. Interestingly, diameters $<2 \mathrm{~nm}$ were measured for most of the nickel nanoparticles (ca. 75\%). This result is quite different from that observed when the nanoparticles were prepared at room temperature using THF as the sole solvent $(2.50 \pm 1.50,25 \% \mathrm{NiNPs}<2 \mathrm{~nm})$ but similar to that in the presence of ethanol at room temperature after prolonged stirring $(1.75 \pm 0.75,78 \%$ NiNPs $<2 \mathrm{~nm})[12 \mathrm{~b}]$. It is noteworthy that the morphology and size distribution was very similar irrespective of the reaction time, as shown in Fig. 1 , recorded at $1,4,8$, and $24 \mathrm{~h}$, respectively. Therefore, the presence of isopropanol at $76{ }^{\circ} \mathrm{C}$ seems to have a beneficial effect in the generation of the nickel nanoparticles as regards their size, uniformity, and stabilisation.

Energy-dispersive X-ray (EDX) analysis on various regions confirmed the presence of nickel, with energy bands centred on 7.5 and $8.3 \mathrm{keV}$ (K lines) and $0.8 \mathrm{keV}$ (L lines) (Fig. 3). The oxygen detected could be attributed to partial oxidation of the nanoparticles during the handling of the sample or to the presence of some residual solvent.

\subsubsection{XPS}

Fig. 4 shows the XPS spectrum of the NiNPs in the presence of isopropanol under an inert argon atmosphere, prior to its utilisation in a hydrogen-transfer reaction. The only peak at $852.2 \mathrm{eV}$ corresponds to the $\mathrm{Ni} 2 \mathrm{p}_{3 / 2}$ level and it is characteristic of $\mathrm{Ni}(0)$ [19]. However, the nanoparticles experienced oxidation upon exposure to air as confirmed by the peak at $856.3 \mathrm{eV}$ (Fig. 5). To determine the unequivocal nature of the oxidised species is beyond the aim of this study. Nonetheless, the peak at 856.3 could be mainly ascribed to the $\mathrm{Ni} 2 \mathrm{p}_{3 / 2}$ level of $\mathrm{Ni}(\mathrm{II})$ in $\mathrm{NiO}$, on the basis of previous studies on the oxidation of nickel nanoparticles in air $[7,20]$.

\subsection{3. $X R D$}

The XRD diffractogram of the NiNPs shows some broad and low intensity peaks (Fig. 6). This behaviour could be attributed to the sample being mainly amorphous and/or to the fact that the crystal domains are $<10 \mathrm{~nm}$. Nevertheless, the peaks corresponding to face-centred cubic nickel could be observed. A high intensity peak at the angle $2 \theta=15.35$ could be assigned to $\mathrm{NiCl}_{2}$. Very probably, the reduction of $\mathrm{NiCl}_{2}$ was not fully completed in this particular experiment of preparation of the NiNPs. The amount of lithium 

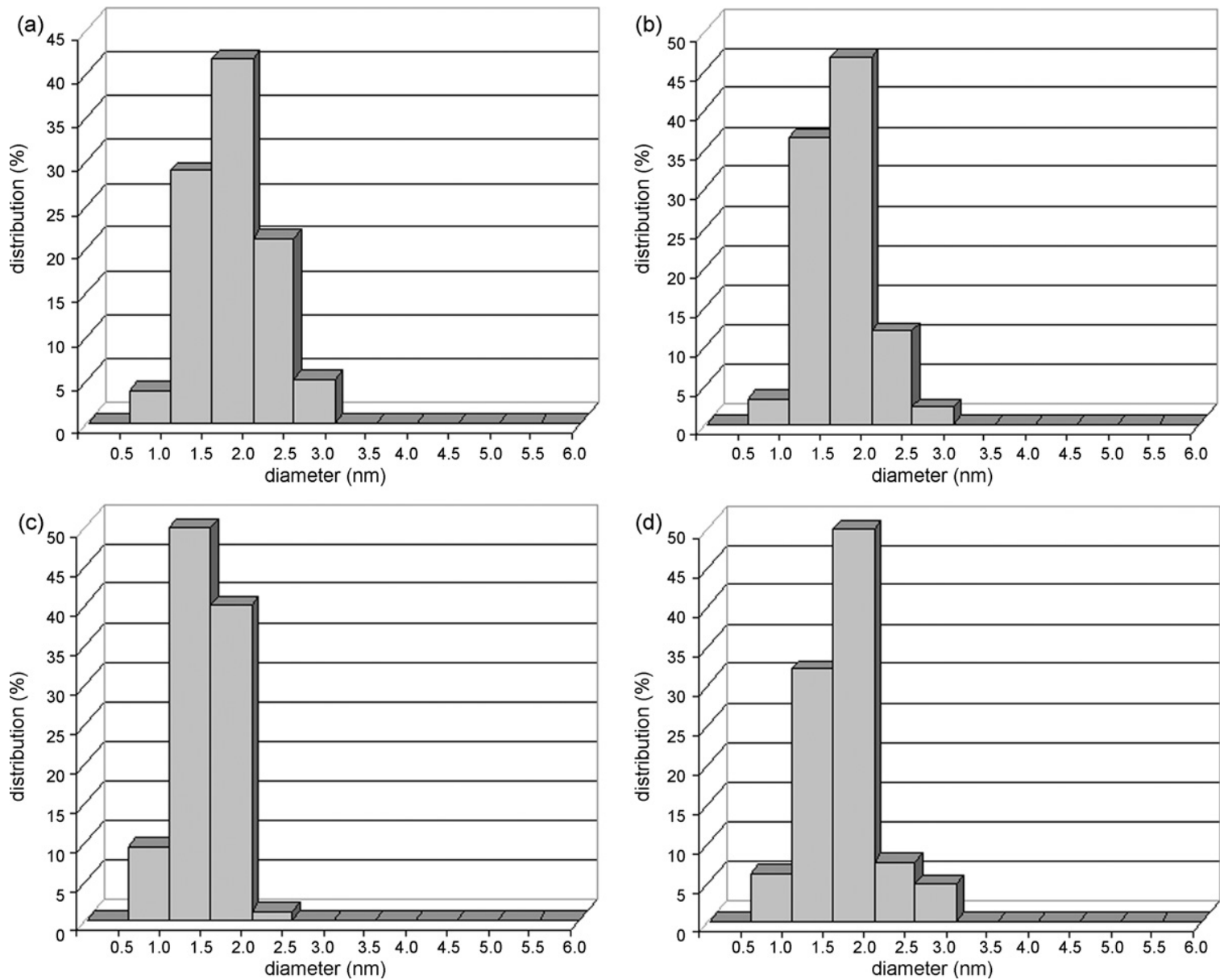

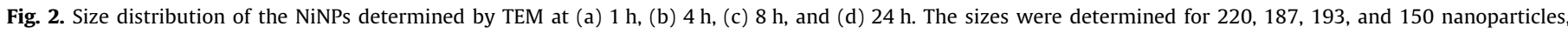
respectively, selected at random.

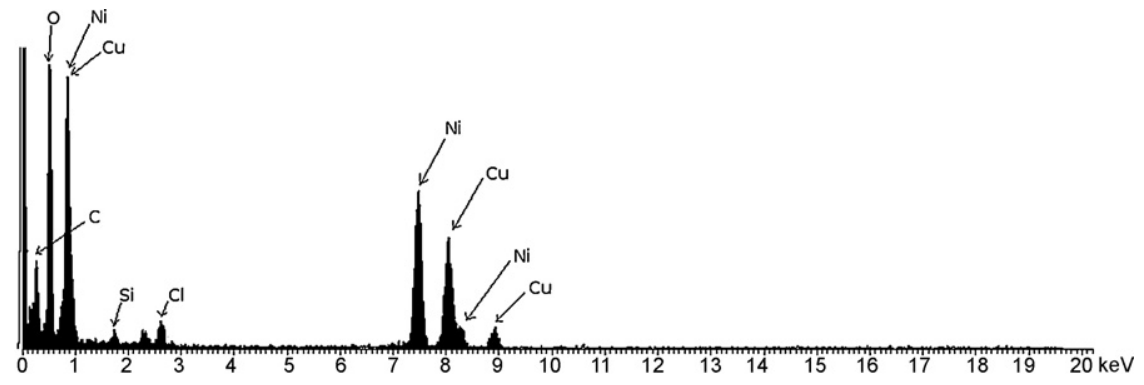

Fig. 3. EDX spectrum of the NiNPs.

metal added to the reaction medium is difficult to weigh accurately (14 mg of fluffy powder in a Schlenk). Consequently, small variations on this weight can completely or partially reduce $\mathrm{NiCl}_{2}$. The fact that $\mathrm{NiCl}_{2}$ was detected by XRD but not by XPS could be due to the fact that $\mathrm{NiCl}_{2}$ is rather insoluble in $\mathrm{THF} / \mathrm{i}$-PrOH at room temperature and tends to settle down in the Schlenk. As a result, droplets of the NiNPs suspension for XPS analysis would be exempt of residual $\mathrm{NiCl}_{2}$, whereas the powder for XRD analysis would contain this $\mathrm{NiCl}_{2}$.

Although the sample preparation was carried out under an inert atmosphere, it was exposed to air during the XRD analysis. The presence of cubic NiO [21], however, was difficult to confirm due to its minor proportion and/or amorphous character. The information and conclusion derived from the XRD analysis are in agreement with the presence of diffuse intensity rings in the selected area electron diffraction pattern (SAED), which also suggest a very small size for the crystalline domains and/or an amorphous character (Fig. 7).

\subsubsection{EPR}

EPR studies on nanosized nickel have been mainly conducted for nickel oxide [22] and supported nickel [23], while its application to unsupported nickel nanoparticles is little documented. We could additionally confirm the presence of NiNPs in the zero-valence state by EPR. The spectra of $\mathrm{NiCl}_{2}$ and commercially available $\mathrm{Ni} / \mathrm{SiO}_{2}-$ $\mathrm{Al}_{2} \mathrm{O}_{3}$ were also recorded as references (Fig. 8). As expected, the blank experiment with $\mathrm{NiCl}_{2}$ gave no paramagnetic signal since in the ground-state electronic configuration all the electrons are matched. A similar magnetic response was observed for the nickel nanoparticles when compared with $\mathrm{Ni} / \mathrm{SiO}_{2}-\mathrm{Al}_{2} \mathrm{O}_{3}$, with the peak-to-peak resonance line width $\left(\Delta H_{\mathrm{pp}}\right)$ being 582 and $1193 \mathrm{G}$, respectively. The 


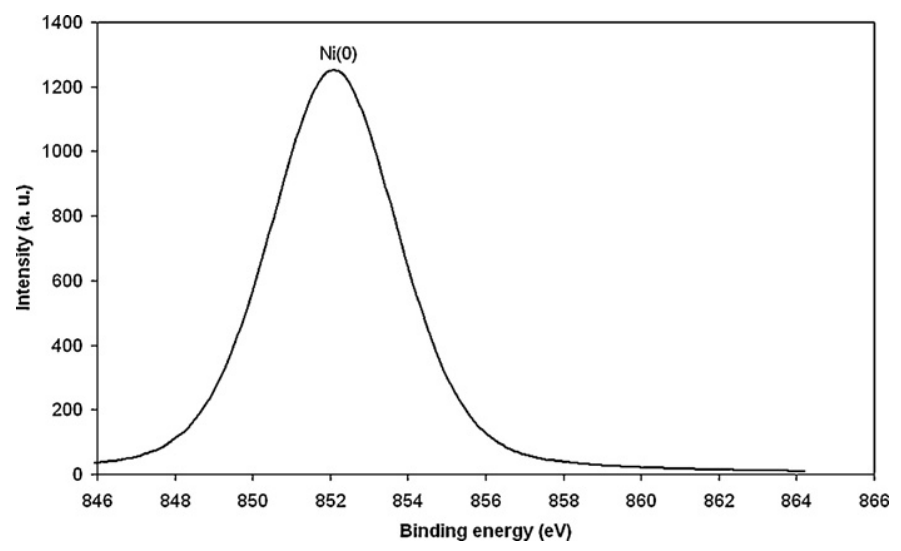

Fig. 4. XPS spectrum of the freshly prepared NiNPs.

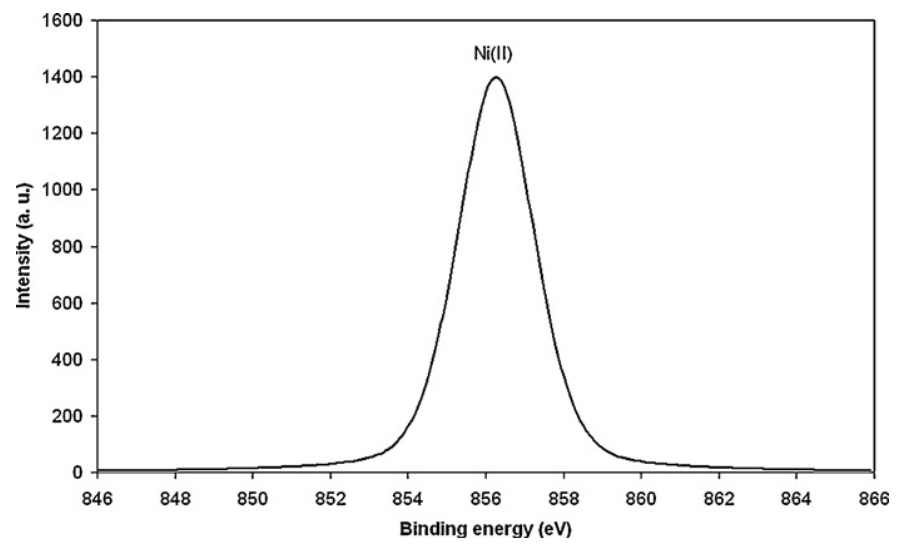

Fig. 5. XPS spectrum of the NiNPs after exposure to air.

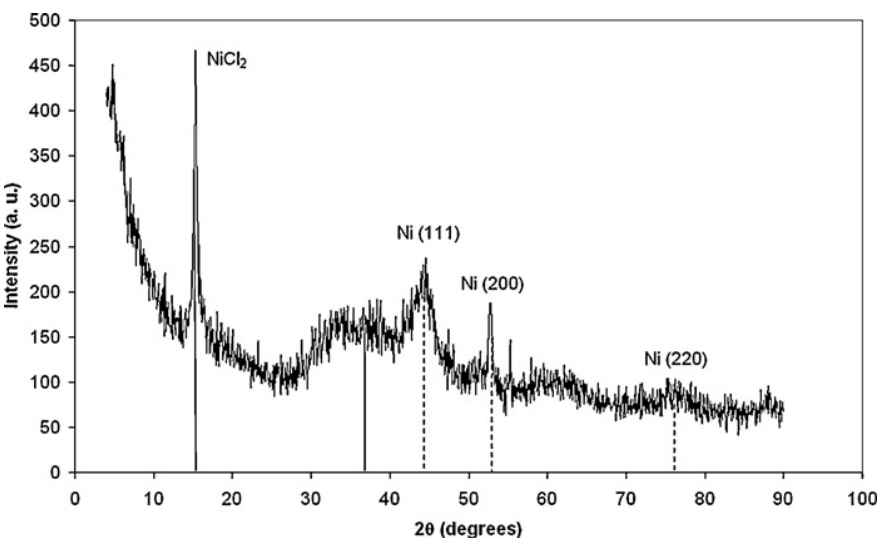

Fig. 6. XRD spectrum of the NiNPs.

effective $g$ factor $\left(g_{\text {eff }}\right)$ was experimentally determined as $h v / \mu_{B} H$, were $v$ is the microwave frequency, $H$ is the magnetic field at which the resonance maximum occurs, $h$ is the Planck's constant, and $\mu_{B}$ is the Bohr magneton. The $g_{\text {eff }}$ values obtained were 2.22 for the NiNPs and 2.15 for the $\mathrm{Ni} / \mathrm{SiO}_{2}-\mathrm{Al}_{2} \mathrm{O}_{3}$ catalyst.

\subsubsection{Surface area}

The surface area of a NiNPs sample was determined to be $33.04 \mathrm{~m}^{2} / \mathrm{g}$. This value is in agreement with those obtained for other metal nanoparticles using the same methodology [24] but greatly differs from that obtained for nickel nanoparticles synthesised from the electric explosion of wire, the specific area of which was $4.405 \mathrm{~m}^{2} / \mathrm{g}$ [7].

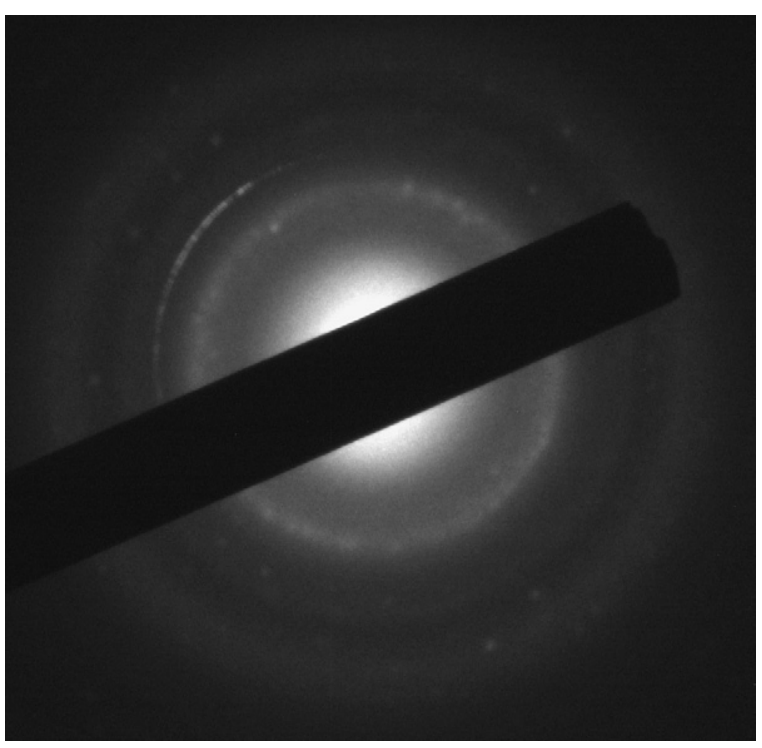

Fig. 7. SAED of the NiNPs.

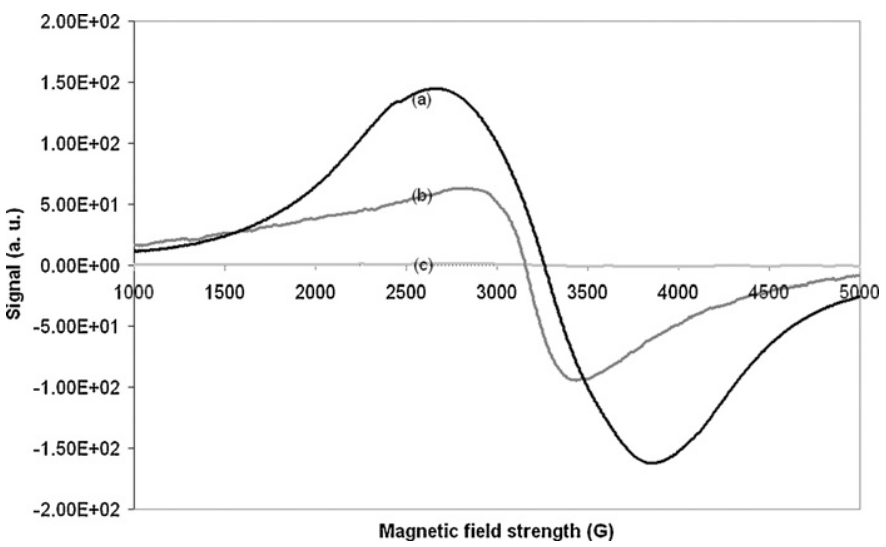

Fig. 8. EPR spectrum of NiNPs: (a) $\mathrm{Ni} / \mathrm{SiO}_{2}-\mathrm{Al}_{2} \mathrm{O}_{3}$, (b) NiNPs, (c) $\mathrm{NiCl}_{2}$.

\subsection{Catalytic and kinetic studies}

All the studies were carried out using acetophenone as a model substrate. In a preliminary study, the transfer hydrogenation was analysed as a function of the reaction temperature (Fig. 9). Low conversion was observed at $25{ }^{\circ} \mathrm{C}$ for short reaction times, which increased up to $54 \%$ after $100 \mathrm{~min}$. The conversion profiles at 40 , 50,60 and $76{ }^{\circ} \mathrm{C}$ show a progressive increment in the slope at short reaction times, with the reaction at $76{ }^{\circ} \mathrm{C}$ reaching $88.5 \%$

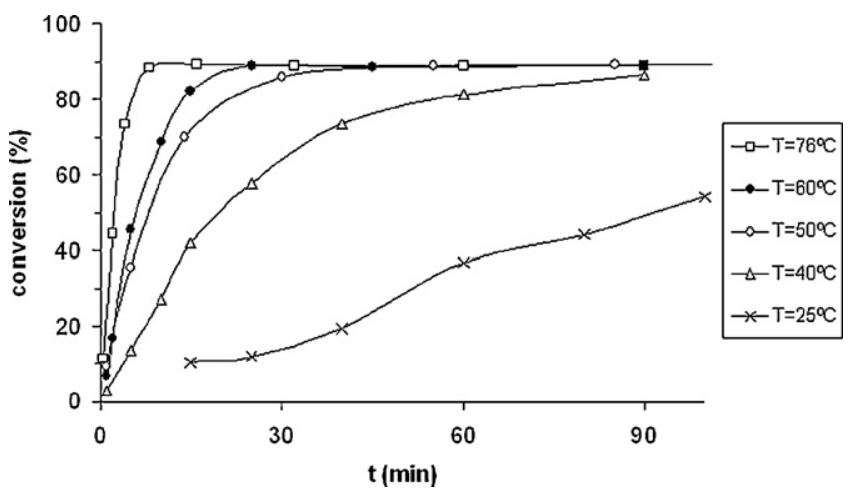

Fig. 9. Transfer hydrogenation of acetophenone profiles at different temperatures. 
Table 1

Transfer hydrogenation of acetophenone with different nickel catalysts.

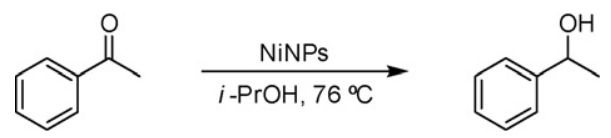

\begin{tabular}{|c|c|c|c|}
\hline Entry & Catalyst & Product & Yield $(\%)^{\mathrm{a}}$ \\
\hline 1 & None & Acetophenone & 100 \\
\hline 2 & $\mathrm{NiNPs}^{\mathrm{b}}$ & Acetophenone & 100 \\
\hline 3 & NiNPs & 1-Phenylethanol & 94 \\
\hline 4 & Raney $\mathrm{Ni}^{\mathrm{C}}$ & Ethylbenzene & 95 \\
\hline 5 & $\mathrm{Ni}-\mathrm{Al}^{\mathrm{c}}$ & Acetophenone & 100 \\
\hline 6 & $\mathrm{Ni} / \mathrm{TiO}_{2}{ }^{\mathrm{d}}$ & Acetophenone & 100 \\
\hline 7 & $\mathrm{Ni} / \mathrm{SiO}_{2}-\mathrm{Al}_{2} \mathrm{O}_{3}{ }^{\mathrm{c}}$ & Acetophenone & 100 \\
\hline 8 & $\mathrm{NiO}^{\mathrm{c}}$ & Acetophenone & 100 \\
\hline
\end{tabular}

a GLC yield.

b In the absence of isopropanol.

c Commercially available catalysts.

d Sample provided by the Inorganic Chemistry Department of the University of Alicante.

conversion in only $8 \mathrm{~min}$ and a stationary conversion of ca. $89 \%$ after 16 min. It is worthwhile mentioning that a similar maximum conversion as that at $76{ }^{\circ} \mathrm{C}$ was recorded for the reactions at 40,50 , and $60{ }^{\circ} \mathrm{C}$ after prolonged heating $(90 \mathrm{~min})$.

We then compared the transfer hydrogenation of acetophenone in the presence of different nickel catalysts at $76{ }^{\circ} \mathrm{C}$ (Table 1 ). Two blank experiments, in the absence of isopropanol (only THF as solvent) and in the absence of nickel gave the unchanged starting material (Table 1, entries 1 and 2, respectively). It is noteworthy that, under the conditions shown in Table 1, only the NiNPs were able to reduce acetophenone to 1 -phenylethanol (Table 1, entry 3 ), whereas Raney Ni led to the hydrogenolysis product ethylbenzene [25]. Some other nickel catalysts were completely inactive in this reaction (Table 1 , entries $5-8$ ).

The concentration effects of acetophenone and isopropanol on the initial rate were also examined. The conversion into 1phenylethanol as a function of time was plotted for a series of reactions carried out with $1 \mathrm{mmol}$ of NiNPs at a constant volume of $2 \mathrm{~mL}$ of THF and $4 \mathrm{~mL}$ of $i-\mathrm{PrOH}$ with $0.25,0.5$ and $1 \mathrm{mmol}$ of acetophenone at $50{ }^{\circ} \mathrm{C}$ (Fig. 10). The initial rates were determined for the individual runs and plotted against the amount of acetophenone. Fig. 10 clearly depicts the reaction rate being dependent upon the amount of acetophenone, albeit a negative slope was obtained. This behaviour could be attributed to the product 1-phenylethanol partially blocking the active sites by adsorption on the nanoparticle surface. We also discovered that high NiNPs/acetophenone molar ratios were crucial for the reaction to occur (Table 2). Thus, 1:1, 1:5 and 0.5:1 NiNPs/ acetophenone molar ratios led to 1-phenylethanol in high yields and short reaction times (Table 2, entries 1, 2, and 6, respectively), whereas no reaction was observed when conducted with 1:20 or 0.25:1 molar ratios (Table 2, entries 5 and 7, respectively) and a modest conversion was obtained with a 1:10 molar ratio after prolonged heating (Table 2, entries 3 and 4).

We next analysed the effects of variation of the isopropanol concentration. In this case, the transfer hydrogenation of acetophenone was conducted with $1 \mathrm{mmol}$ of NiNPs, $1 \mathrm{mmol}$ of acetophenone, $2 \mathrm{~mL}$ of THF and 1, 4, 6, and $8 \mathrm{~mL}$ of isopropanol at $50{ }^{\circ} \mathrm{C}$ (Fig. 11). The graphic showing the initial rate against the amount of isopropanol unveiled an unexpected behaviour. Thus, although the highest initial rate was recorded for the largest amount of isopropanol $(8 \mathrm{~mL})$, both lower conversions and initial rate were obtained with $4 \mathrm{~mL}$ in comparison with those obtained with $1 \mathrm{~mL}$ of isopropanol. Recently, Adolfsson et al. studied the
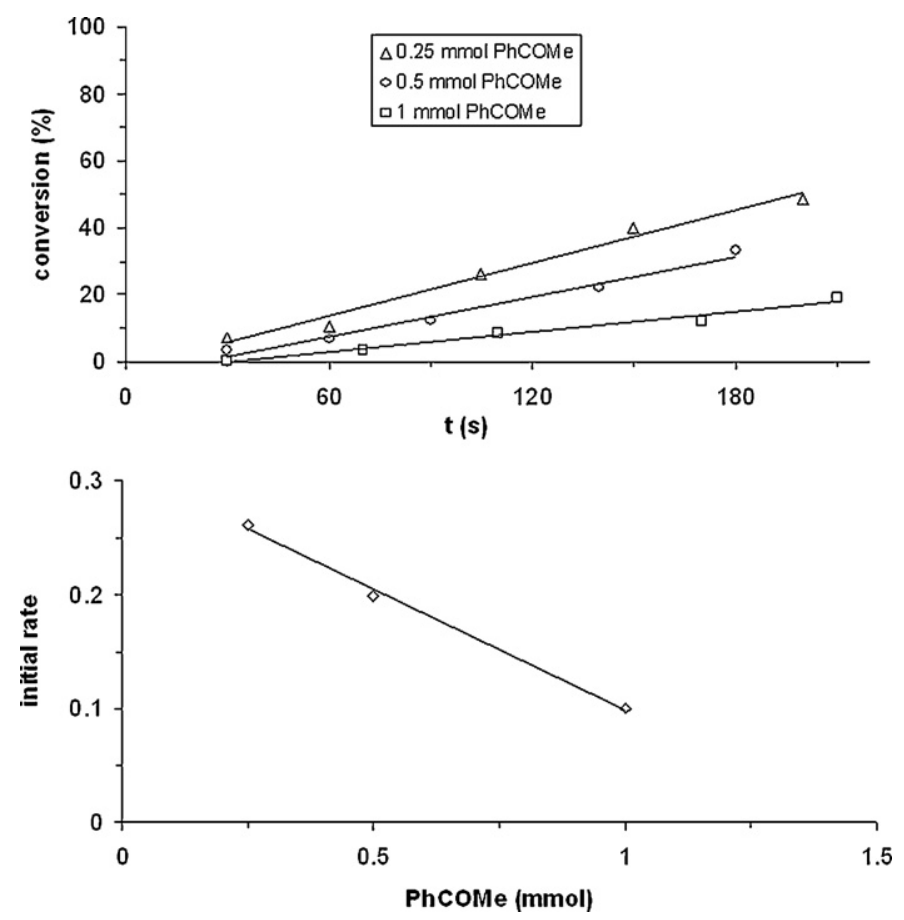

Fig. 10. Conversion and initial rate as a function of the amount of acetophenone.

Table 2

Transfer hydrogenation of acetophenone at different NiNPs/acetophenone ratios.

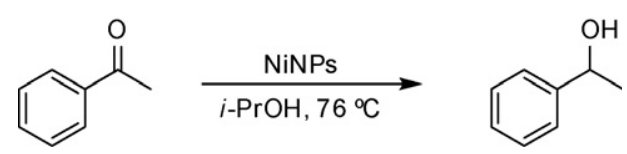

\begin{tabular}{llcc}
\hline Entry & NiNPs/acetophenone $(\mathrm{mmol})$ & $t(\mathrm{~h})$ & ${\text { Yield }(\%)^{\mathrm{a}}}^{-}$ \\
\hline 1 & $1: 1$ & 0.13 & 89 \\
2 & $1: 5$ & 1 & 87 \\
3 & $1: 10$ & 1 & 7 \\
4 & $1: 10$ & 30 & 36 \\
5 & $1: 20$ & 24 & 0 \\
6 & $0.5: 1$ & 2 & 86 \\
7 & $0.25: 1$ & 24 & 0 \\
\hline
\end{tabular}

a GLC yield.

homogeneous ruthenium-pseudodipeptide catalysed asymmetric transfer hydrogenation of ketones using $i$-PrONa $/ i-\mathrm{PrOH}$ and also observed that the initial rate was not directly proportional to the hydrogen donor amount [26]. In both cases, a large excess of isopropanol was utilised with respect to acetophenone.

In the same report as above, Adolfsson described an increase in the degree of conversion after the addition of $\mathrm{LiCl}$ [26]. The authors proposed that hydride transfer from isopropanol occurred in a Meerwein-Ponndorf-Verley-type fashion [27] (i.e., direct hydrogen transfer through a six-membered transition state composed of the hydrogen acceptor, metal, and hydrogen donor, without the participation of metal hydride intermediates) but involving ruthenium monohydride species. In our case, $\mathrm{LiCl}$ is in situ generated during the formation of the nickel nanoparticles. Previously performed deuteration experiments [14a], however, indicated the participation of a dihydride-type mechanism, in which the two hydrogens of the donor become equivalent after being transferred to the metal to give the dihydride. These results, together with the fact that the reaction rate was found to be 

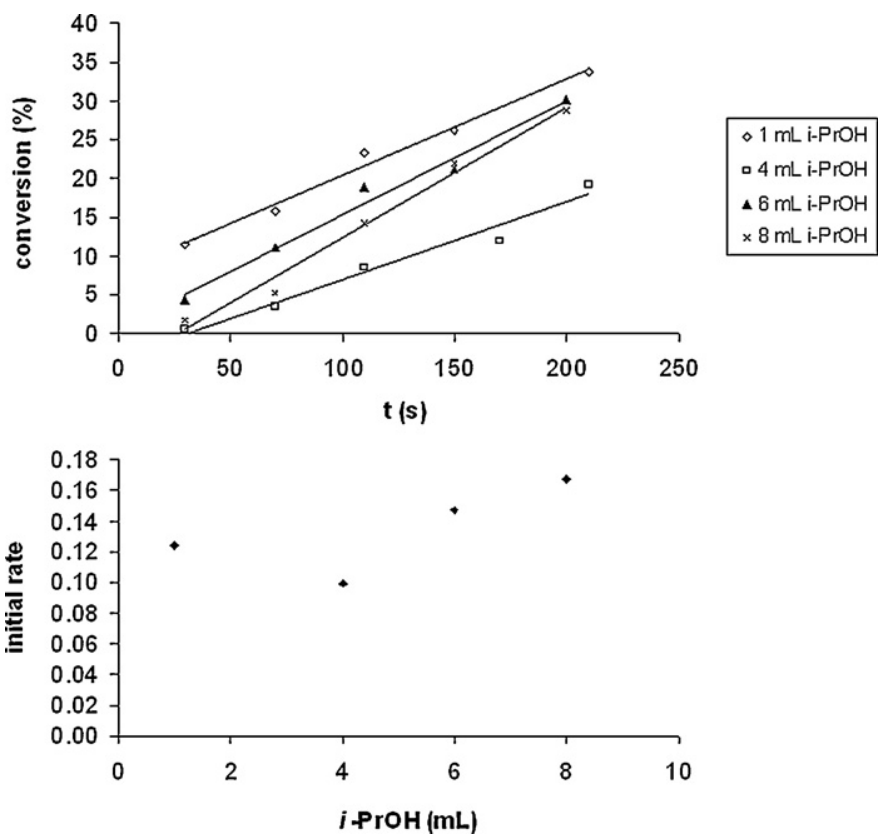

Fig. 11. Conversion and initial rate as a function of the amount of isopropanol. (a)

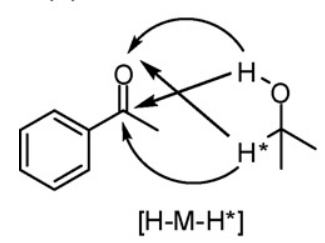
-acetone 然<smiles>C[C@@H](O)c1ccccc1</smiles>
$(50 \%)$ (b)<smiles>CC(=O)c1ccccc1</smiles><smiles>CC(C)(C)[N+](=O)[O-]</smiles><smiles>CC(O)(CS(=O)(=O)O)c1ccccc1</smiles>

Scheme 1. (a) The dihydride-type mechanism for the transition-metal catalysed transfer hydrogenation with isopropanol. (b) Deuterium labelling experiment in the transfer hydrogenation of acetophenone.

independent of the $\mathrm{LiCl}$ concentration, allows concluding that $\mathrm{LiCl}$ is a spectator in our transfer hydrogenation reaction (Scheme 1).

\subsection{Catalyst deactivation}

One main advantage of the NiNPs in the transfer hydrogenation of carbonyl compounds was their reuse capability. Thus, in the stoichiometric version (1:1 NiNPs/acetophenone ratio), the NiNPs could be reutilised over four consecutive cycles without any apparent loss of activity ( $94 \%$ average yield), the latter decreasing up to the seventh cycle (59\% yield) [14a]. In the sub-stoichiometric version (1:5 NiNPs/acetophenone ratio, $20 \mathrm{~mol} \% \mathrm{Ni}$ ), the NiNPs could be reutilised three times without any apparent loss of activity (88\% average yield) with a final fifth cycle (77\% yield) [14b]. We were intrigued by this gradual loss of activity but, even more, by the total deactivation after the above-mentioned cycles. The physical deposition of matter and/or strong chemisorption of species can induce important surface modification leading to deactivation [28]. In particular, coke formation can occur by catalytic decomposition of organic compounds even at low temperatures [29]. The chemical nature of this deposit depends

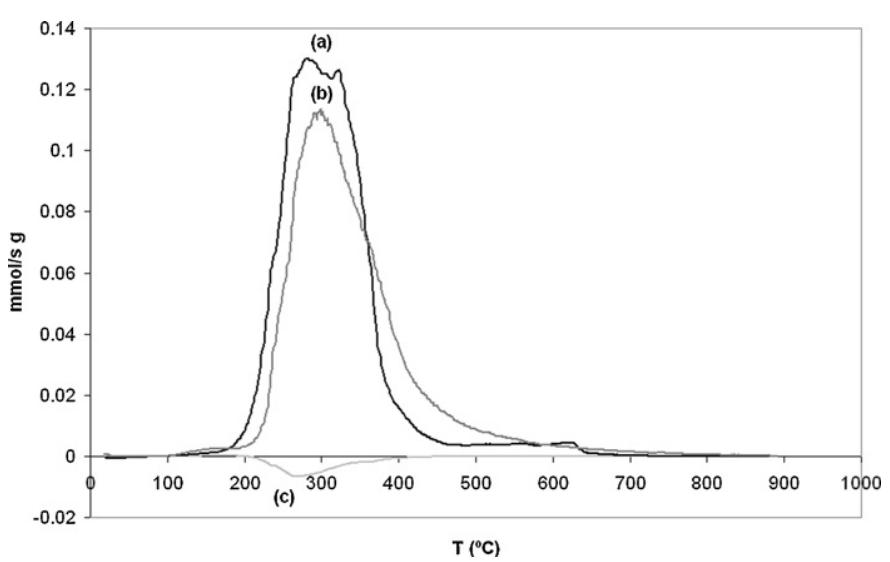

Fig. 12. TPO profile of deactivated NiNPs: (a) $\mathrm{CO}_{2}$, (b) $\mathrm{H}_{2} \mathrm{O}$, (c) $\mathrm{CO}$.

on the feedstock molecules, which react to form coke. The deactivation of some common heterogeneous hydrogenation catalysts (e.g., Pd/C and Raney $\mathrm{Ni}$ ) after aging them in alcoholic solvents has been recently reported [30]. Alcohols such as methanol, ethanol, 1-propanol, and 2-propanol got decomposed into carbon monoxide and carbonaceous species with the consequent catalyst surface poisoning. Sinterisation, agglomeration or size increase of the nanoparticles can also affect their catalytic activity and, therefore, must also be analysed.

In order to know whether the deactivation of the NiNPs in hydrogen-transfer reactions was due to the formation of coke, a deactivated sample, after reutilisation in the transfer hydrogenation of acetophenone with isopropanol, was analysed by temperature programmed oxidation (TPO), Raman spectroscopy and thermogravimmetry (TG).

TPO experiments have been found to be an efficient tool to evaluate the presence of carbonaceous materials [31], either derived from the support [32] or from a chemical reaction [33]. The TPO profiles of the deactivated NiNPs in Fig. 12 show that $\mathrm{CO}_{2}$ formation starts at $200{ }^{\circ} \mathrm{C}$ with a maximum production at 270 $320^{\circ} \mathrm{C}$. These data rule out the possible $\mathrm{CO}_{2}$ emission from coke combustion, which typically starts above $400{ }^{\circ} \mathrm{C}$ [34]. A similar profile to that of $\mathrm{CO}_{2}$ is observed for $\mathrm{H}_{2} \mathrm{O}$ with a rate of formation $<1.3 \times 10^{-4} \mathrm{mmol} \mathrm{s}^{-1} \mathrm{~g}^{-1}$. Therefore, organic compounds and molecular hydrogen, derived from the transfer hydrogenation reaction or sample preparation and adsorbed on the catalyst surface, could account for the results observed.

Raman spectroscopy has been widely applied to the study of used catalysts [35]. It is known that the precise absorptions of coke strongly depend on the compounds present in the reaction medium and deactivation conditions. A typical Raman spectrum of graphite or coke exhibits two bands: the first one is more intense and located at about $1590 \mathrm{~cm}^{-1}$, and a second one, normally less intense and broader centred at about $1355 \mathrm{~cm}^{-1}[35,36]$. The NiNPs showed a rather flat Raman spectrum (Fig. 13) either before or after their deactivation. The bands at a lower frequency are present in both the fresh and deactivated samples, and correspond to $\mathrm{NiCl}_{2}$ (ca. 175 and $270 \mathrm{~cm}^{-1}$ ) and $\mathrm{NiO}$ (ca. $500 \mathrm{~cm}^{-1}$ ). The presence of small amounts of $\mathrm{NiCl}_{2}$ could be attributed to an incomplete reduction during the formation of the NiNPs, whereas partial oxidation of the NiNPs during the sample preparation, involving filtration and washing in air, could account for the formation of $\mathrm{NiO}$.

Thermogravimetric analysis of a deactivated sample of the NiNPs did not show any significant difference with respect to that of freshly prepared NiNPs in the temperature range applied (room temperature to $700{ }^{\circ} \mathrm{C}$ ). 


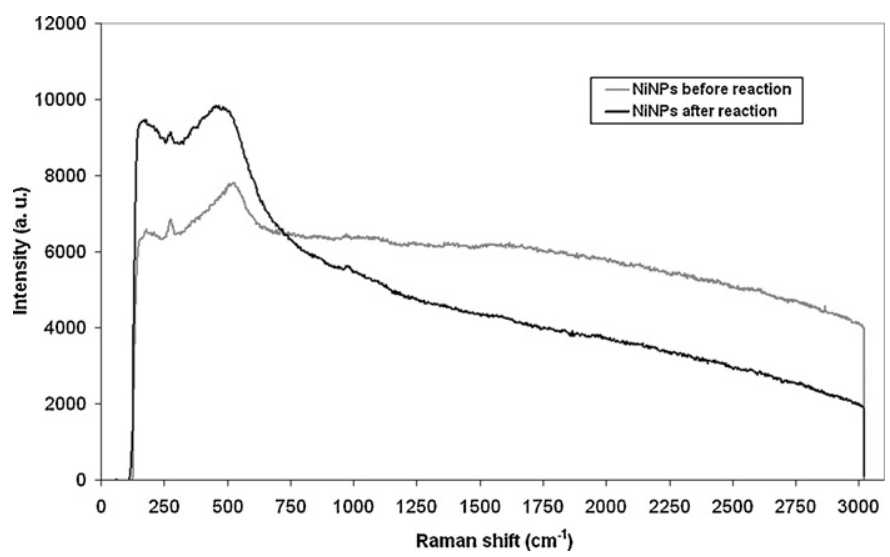

Fig. 13. Raman spectrum before and after deactivation of the NiNPs.

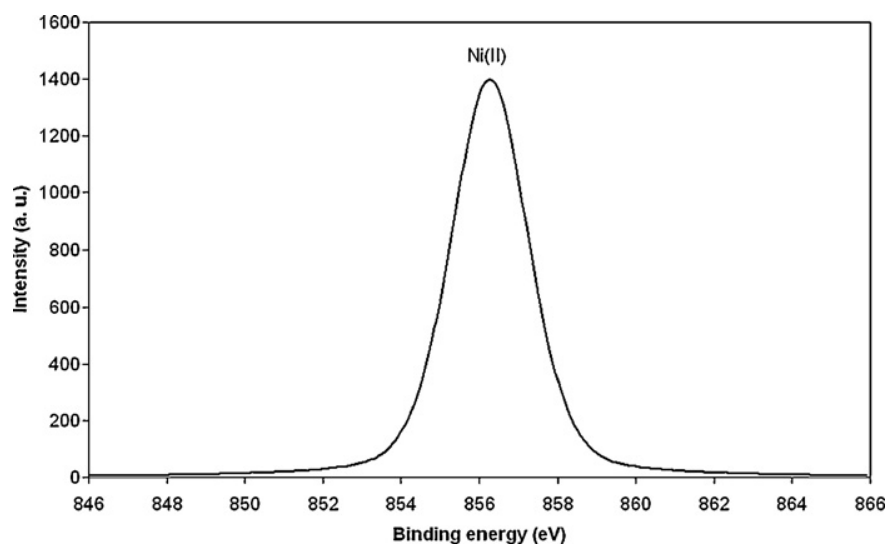

Fig. 14. XPS spectrum of deactivated NiNPs after reuse.

From the above experiments, we can conclude that the deactivation of the catalyst after reuse is not due to coke formation during the reaction, but more plausibly, to partial oxidation to nickel(II) oxide. In fact, different deactivated samples examined by XPS after reuse showed only one peak at $856.3 \mathrm{eV}$, corresponding to the $\mathrm{Ni} 2 \mathrm{p}_{3 / 2}$ level of $\mathrm{Ni}(\mathrm{II})$ (Fig. 14). Although all the reactions were performed under an inert atmosphere, isopropanol was not exempt of oxygen when used in each cycle. Therefore, a certain degree of surface oxidation could definitely stop the catalytic activity of the NiNPs.

\subsection{Nature of the catalyst}

Catalysis by metal nanoparticles can be considered a kind of "semi-heterogeneous catalysis", at the frontier between homogeneous and heterogeneous catalysis [18]. Therefore, it is important to determine whether the NiNPs are the true catalysts or a reservoir for metal atoms that leach into solution [37]. In this section, we will describe a series of control experiments in order to know the true nature of the catalytically active species [38].

TEM analysis is a tool for the direct observation of NPs as heterogeneous species present in the reaction medium. The formation of NiNPs could be confirmed by TEM for every hydrogen-transfer reaction practiced.

In the previously described catalytic studies the NiNPs manifested some activity since the very beginning and, as expected, no induction period was observed in the kinetic profiles, this behaviour being more typical for NPs generated from a homogeneous catalyst.
Table 3

Catalyst poisoning experiments in the transfer hydrogenation of acetophenone.

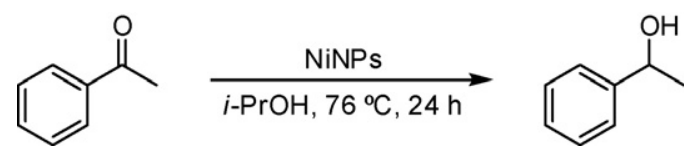

\begin{tabular}{|c|c|c|c|c|}
\hline Entry & y Acetophenone/NiNPs (equiv.) & ) Poison (equiv.) & ) Product & Yield $(\%)^{a}$ \\
\hline 1 & $5: 1$ & $\mathrm{Hg}(5)$ & 1-Phenylethanol & 10 \\
\hline 2 & $1: 1$ & $\mathrm{Hg}(5)$ & 1-Phenylethanol & 10 \\
\hline 3 & $5: 1$ & $\mathrm{Hg}(10)$ & Acetophenone & 100 \\
\hline 4 & $1: 1$ & $\mathrm{Hg}(10)$ & Acetophenone & 100 \\
\hline 5 & $5: 1$ & $\mathrm{PPh}_{3}(1)$ & Acetophenone & 100 \\
\hline 6 & $5: 1$ & $\mathrm{PPh}_{3}(0.5)$ & Acetophenone & 100 \\
\hline 7 & $5: 1$ & $\mathrm{PPh}_{3}(0.25)$ & Acetophenone & 100 \\
\hline 8 & $1: 1$ & $\mathrm{PPh}_{3}(1)$ & Acetophenone & 100 \\
\hline 9 & $1: 1$ & $\mathrm{PPh}_{3}(0.5)$ & Acetophenone & 100 \\
\hline 10 & $1: 1$ & $\mathrm{PPh}_{3}(0.25)$ & Acetophenone & 100 \\
\hline
\end{tabular}

${ }^{a}$ GLC yield.

\subsubsection{Catalyst poisoning experiments}

The mercury test has been largely exploited to identify heterogeneous catalysts due to its ability to poison metal $(0)$ heterogeneous catalysts by formation of amalgam or adsorption on the metal surface [38]. In fact, mercury $(0)$ is probably the most effective in poisoning metals that form an amalgam, such as Pt, Pd, and $\mathrm{Ni}$ [39]. When acetophenone was subjected to the transfer hydrogenation, in the presence of 20 or $100 \mathrm{~mol} \%$ NiNPs and 5 equiv. $\mathrm{Hg}(0), 10 \%$ of the reduced product 1 -phenylethanol was obtained (Table 3, entries 1 and 2, respectively). By using double amount of $\mathrm{Hg}(0)$, however, the reaction was completely inhibited (Table 3, entries 3 and 4). Since mercury has been reported to occasionally induce side reactions, the results of the tests are not always conclusive enough to know the catalyst nature. Therefore, it is convenient to confirm the poisoning results with a second test. $\mathrm{PPh}_{3}$ can also be used as a poison due to its strong affinity to metal centres. If a catalyst is poisoned completely with $<1$ equiv. of $\mathrm{PPh}_{3}$, that evidences the presence of a heterogeneous catalyst. Six different experiments were carried out by varying the acetophenone/NiNPs ratio and the amount of $\mathrm{PPh}_{3}$. We observed that irrespective the acetophenone/NiNPs ratio, the transfer hydrogenation was inhibited either by stoichiometric or sub-stoichiometric amounts of $\mathrm{PPh}_{3}$ (Table 3, entries 5-10).

\subsubsection{Filtration test $[37,38]$}

This test relies on a comparison of the catalytic activity before and after filtering the active catalyst solution. For this purpose, a standard NiNPs black suspension, prepared from $\mathrm{NiCl}_{2}, \mathrm{Li}$, and DTBB (cat.) in THF $/ i$-PrOH at $76^{\circ} \mathrm{C}$, was used. When this suspension was filtered though a pad containing Celite, the resulting filtrate was found to be inactive in the transfer hydrogenation of acetophenone. In general, membrane filters with pores small enough to exclude nanoparticles are slow and difficult to use. Therefore, we attempted filtration of the above suspension via syringe through a commercially available $0.45 \mu \mathrm{m}$ pore size filter under argon. The resulting colourless filtrate was analysed by ICPMS showing the presence of $64 \mathrm{ppb} \mathrm{Ni}$. This filtrate was shown to be inactive when subjected to the transfer hydrogenation of acetophenone (Scheme 2). The low content of nickel in the filtrate could be due to nanoparticle agglomeration during the transfer of the NiNPs suspension with the syringe. In fact, any reaction carried out in a flask different to that in which the NiNPs were generated, and to which part of the original suspension was transferred via a syringe, failed. 


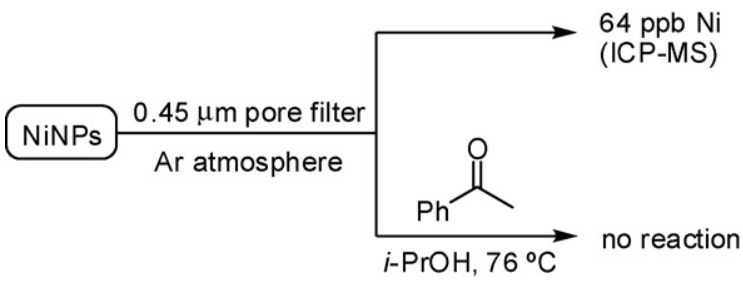

Scheme 2. Filtration test in the transfer hydrogenation of acetophenone.

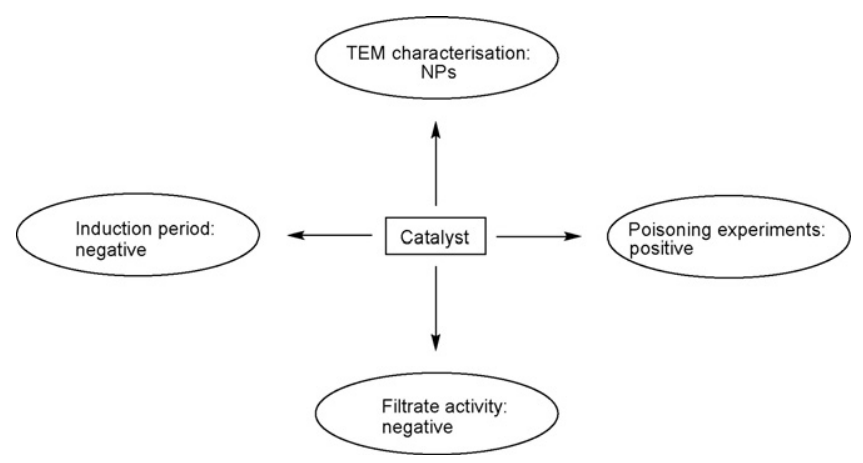

Scheme 3. Summary of the tests to determine the nature of the catalysis.

From the essays above it can be inferred that the transfer hydrogenation of carbonyl compounds catalysed by NiNPs occur under heterogeneous catalysis on the surface of the NiNPs (Scheme 3).

\section{Conclusion}

Nickel nanoparticles, generated by reduction of nickel(II) chloride with lithium in the presence of a catalytic amount of DTBB and utilised in the transfer hydrogenation of carbonyl compounds with isopropanol, have been characterised by TEM, EDX, XPS, XRD, EPR and BET area. The fastest conversion was reached at $76{ }^{\circ} \mathrm{C}(88.5 \%$ in $8 \mathrm{~min}$ ). The NiNPs have been found to be superior to commercially available catalysts, including Raney nickel. The reaction rate is dependent upon the amount of acetophenone with a negative slope, but it is not proportional to the amount of isopropanol, and independent on the $\mathrm{LiCl}$ concentration. Based on TPO experiments, Raman spectroscopy, TG and XPS analysis, the deactivation of the catalyst with reiterative reuse has been ascribed to surface oxidation, while the formation of coke has been ruled out. Moreover, the NiNPs have been demonstrated to be the true catalyst in this reaction, the heterogeneous nature of the process being unequivocally established on the basis of TEM, kinetic, poisoning, and filtration experiments.

\section{Acknowledgements}

This work was generously supported by the Spanish Ministerio de Educación y Ciencia (MEC; grant no. CTQ2007-65218 and Consolider Ingenio 2010-CSD2007-00006) and the Generalitat Valenciana (grant no. PROMETEO/2009/039). P. R. thanks the MEC for a predoctoral grant. We are grateful to Javier Ruiz-Martínez for carrying out the TPO analysis at the Inorganic Chemistry Department of the University of Alicante.

\section{References}

[1] W. Tseng, C. Chen, J. Mater. Sci. 41 (2006) 1213-1219.

[2] (a) T. Hyeon, Chem. Commun. (2003) 927-934;

(b) S.P. Gubin, Y.A. Koksharov, G.B. Khomutov, G.Y. Yurkov, Russ. Chem. Rev. 74 (2005) 489-520.
[3] R. Karmhag, T. Tesfamichael, E. Wäckelgård, G. Nikalsson, M. Nygren, Sol. Energy 68 (2000) 329-333.

[4] (a) I.S. Lee, N. Lee, S.R. Paik, T. Hyeon, J. Am. Chem. Soc. 46 (2007) 4630-4660; (b) S. Rodríguez-Llamazares, J. Merchán, I. Olmedo, H.P. Marambio, J.P. Muñoz, P. Jara, J.C. Sturm, B. Chornik, O. Peña, N. Yutronic, M.J. Kogan, J. Nanosci. Nanotechnol. 8 (2008) 3820-3827.

[5] J. Geng, B.F.G. Johnson, in: B. Zhou, S. Hermans, G.A. Somorjai (Eds.), Nanotechnology in Catalysis, vol. 1, Springer, Berlin, 2004, pp. 159-182.

[6] (a) A. Houdayer, R. Schneider, D. Billaud, J. Ghanbaja, J. Lambert, Synth. Met. 151 (2005) 165-174;

(b) J. Park, E. Kang, S.U. Son, H.M. Park, M.K. Lee, J. Kim, K.W. Kim, H.-J. Noh, J.-H. Park, C.J. Bae, J.-G. Park, T. Hyeon, Adv. Mater. 17 (2005) 429-434;

(c) M. Kidwai, N.K. Mishra, V. Bansal, A. Kumar, S. Mozumdar, Catal. Commun. 9 (2008) 612-617;

(d) A. Saxena, A. Kumar, S. Mozumdar, Appl. Catal. A: Gen. 317 (2007) 210-215; (e) A. Dhakshinamoorthy, K. Pitchumani, Tetrahedron Lett. 49 (2008) 18181823

(f) V. Polshettiwar, B. Baruwati, R.S. Varma, Green Chem. 11 (2009) 127-131.

[7] P. Song, D. Wen, Z.X. Guo, T. Korakianitis, Phys. Chem. Chem. Phys. 10 (2008) 5057-5065.

[8] (a) L.K. Kurihara, G.M. Chow, P.E. Schoen, Nanostruct. Mater. 5 (1995) 607-613; (b) Y. Wada, H. Kuramoto, T. Sakata, H. Mori, T. Sumida, T. Kitamura, S. Yanagida, Chem. Lett. (1999) 607-608;

(c) M. Tsuji, M. Hashimoto, T. Tsuji, Chem. Lett. (2002) 1232-1233;

(d) L. Bai, J. Fan, Y. Cao, F. Yuan, A. Zuo, Q. Tang, J. Cryst. Growth 311 (2009) $2474-$ 2479.

[9] (a) D.-H. Chen, S.-H. Wu, Chem. Mater. 12 (2000) 1354-1360;

(b) S.-H. Wu, D.-H. Chen, J. Colloid Interface Sci. 259 (2003) 282-286;

(c) S.-H. Wu, D.-H. Chen, Chem. Lett. (2004) 406-407;

(d) K.H. Kim, Y.B. Lee, S.G. Lee, H.C. Park, S.S. Park, Mater. Sci. Eng., A 381 (2004) 337-342.

[10] (a) G.G. Couto, J.J. Klein, W.H. Schreiner, D.H. Mosca, A.J.A. de Oliveira, A.J.G. Zarbin, J. Colloid Interface Sci. 311 (2007) 461-468;

(b) P.K. Khanna, P.V. More, J.P. Jawalkar, B.G. Bharate, Mater. Lett. 63 (2009) 1384-1386

[11] (a) F. Alonso, G. Radivoy, M. Yus, Russ. Chem. Bull., Int. Ed. 52 (2003) 2576;

(b) F. Alonso, M. Yus, Chem. Soc. Rev. 33 (2004) 284-293;

(c) F. Alonso, M. Yus, Pure Appl. Chem. 80 (2008) 1005-1012.

[12] (a) F. Alonso, J.J. Calvino, I. Osante, M. Yus, Chem. Lett. 34 (2005) 12621263;

(b) F. Alonso, J.J. Calvino, I. Osante, M. Yus, J. Exp. Nanosci. 1 (2006) 419433.

[13] (a) F. Alonso, I. Osante, M. Yus, Adv. Synth. Catal. 348 (2006) 305-308;

(b) F. Alonso, I. Osante, M. Yus, Synlett (2006) 3017-3020;

(c) F. Alonso, I. Osante, M. Yus, Tetrahedron 63 (2007) 93-102;

(d) F. Alonso, P. Riente, M. Yus, ARKIVOC iv (2008) 8-15.

[14] (a) F. Alonso, P. Riente, M. Yus, Tetrahedron 64 (2008) 1847-1852;

(b) F. Alonso, P. Riente, M. Yus, Tetrahedron Lett. 49 (2008) 1939-1942;

(c) F. Alonso, P. Riente, M. Yus, Tetrahedron 65 (2009) 10637-10643.

[15] F. Alonso, P. Riente, M. Yus, Synlett (2008) 1289-1292.

[16] (a) F. Alonso, P. Riente, M. Yus, Synlett (2007) 1877-1880;

(b) F. Alonso, P. Riente, M. Yus, Eur. J. Org. Chem. (2008) 4908-4914.

[17] (a) F. Alonso, P. Riente, M. Yus, Synlett (2009) 1579-1582;

(b) F. Alonso, P. Riente, M. Yus, Tetrahedron Lett. 50 (2009) 3070-3073:

(c) F. Alonso, P. Riente, M. Yus, Eur. J. Org. Chem (2009) 6034-6042.

[18] D. Astruc, F. Lu, J.R. Aranzaes, Angew. Chem. Int. Ed. 44 (2005) 7852-7872.

[19] R.P. Furstenau, G. McDougall, M.A. Langell, Surf. Sci. 150 (1985) 55-79.

[20] S. Lee, N. Lee, J. Park, B.H. Kim, Y.-W. Yi, T. Kim, T.K. Kim, I.H. Lee, S.R. Paik, T Hyeon, J. Am. Chem. Soc. 128 (2006) 10658-10659.

[21] C.M.R. Remédios, J.M. Sasaki, Powder Diffr. 23 (2008) S56-S58.

[22] H. Shim, P. Dutta, M.S. Seehra, J. Bonevich, Solid State Commun. 145 (2008) 192196.

[23] (a) S. Lefondeur, S. Monteverdi, S. Molina, M.M. Bettahar, Y. Fort, E.A. Zhilinskaya, A. Aboukais, M. Lelaurain, J. Mater. Sci. 36 (2001) 2633-2638;

(b) A.A. Konchits, F.V. Motsnyi, Y.N. Petrov, S.P. Kolesnik, V.S. Yefanov, M.L. Terranova, E. Tamburri, S. Orlanducci, V. Sessa, M. Rossi, J. Appl. Phys. 100 (2006) $124315 / 1-124315 / 7$

(c) N. Guskos, M. Maryaniak, J. Typek, P. Podsiadly, U. Narkiewicz, E. Senderek, Z. Roslaniec, J. Non-Cryst. Solids 355 (2009) 1400-1404.

[24] Y. Moglie, Doctoral Thesis Dissertation, Bahía Blanca, Argentina, 2009.

[25] M.J. Andrews, C.N. Pillai, Indian J. Chem. B 16 (1978) 465-468.

[26] J. Wettergren, E. Buitrago, P. Ryberg, H. Adolfsoon, Chem. Eur. J. 15 (2009) 57095718.

[27] (a) C.F. de Graauw, J.A. Peters, H. van Bekkum, J. Huskens, Synthesis (1994) 10071017

(b) K. Nishide, M. Node, Chirality 14 (2002) 759-767;

(c) J.S. Cha, Org. Proc. Res. Dev. 10 (2006) 1032-1053.

[28] J.J. Spivey, G.W. Roberts, B.H. Davies (Eds.), Catalyst Deactivation, Elsevier, Amsterdam, 2001.

[29] (a) A. Shamsi, Appl. Catal. A: Gen. 277 (2004) 23-30

(b) Y.-X. Pan, C.-J. Liu, P. Shi, J. Power Sources 176 (2008) 46-53.

[30] U.K. Singh, S.W. Krska, Y. Sun, Org. Process Res. Dev. 10 (2006) 1153-1156.

[31] C. Li, T.C. Brown, Carbon 39 (2000) 725-732.

[32] See, for instance: E. Ochoa-Fernández, D. Chen, Z. Yu, B. Tøtdal, M. Rønning, A. Holmen, Catal. Today, 102/103 (2005) 45-49.

[33] A. Tanksale, J.N. Beltramini, J.A. Dumesic, G.Q. Lu, J. Catal. 258 (2008) 366-377. 
[34] (a) Y. Rezgui, M. Guemini, Ing. Eng. Chem. Res. 47 (2008) 4056-4060; (b) A. Benamar, Z. Bechket, Y. Boucheffa, A. Miloudi, C. R. Chim. 12 (2009) 706715.

[35] (a) J. Li, G. Xiong, Z. Feng, Z. Liu, Q. Xin, C. Li, Micropor. Mesopor. Mater. 39 (2000) 275-280;

(b) Y.T. Chua, P.C. Stair, J. Catal. 213 (2003) 39-46.

[36] (a) M. Digne, K. Marchand, P. Bourges, Oil Gas Sci. Technol.: Rev. IFP 62 (2007) 91-99;

(b) G. Katumba, B.W. Mwakikunga, T.R. Mothibinyane, Nanoscale Res. Lett. 3 (2008) 421-426.
[37] (a) A.V. Gaikwad, A. Holuigue, M.B. Thathagar, J.E. ten Elshof, G. Rothenberg, Chem. Eur. J. 13 (2007) 6908-6913;

(b) L. Durán Pachón, G. Rothenberg, Appl. Organomet. Chem. 22 (2008) 288-299.

[38] (a) J.A. Widegren, R.G. Finke, J. Mol. Catal. A: Chem. 198 (2003) 317-341;

(b) N.T.S. Phan, M. Van der Sluys, C.W. Jones, Adv. Synth. Catal.348 (2006)609-679;

(c) M. Gómez, I. Favier, in: B. Corain, G. Schmid, N. Toshima (Eds.), Metal Nanoclusters in Catalysis and Materials Science: The Issue of Size Control, Elsevier, Amsterdam, 2008(chapter 31).

[39] (a) K.C. Campbell, J.S. Hislop, J. Catal. 13 (1969) 12-19;

(b) J. Dunleavy, Platinum Metals Rev. 50 (2006) 156.

Please cite this article in press as: F. Alonso, et al., Appl. Catal. A: Gen. (2010), doi:10.1016/j.apcata.2010.01.044 